\title{
Effect of INM on Growth and Yield Attributes of Dill Seed (Anethum graveolens L.) Under North Gujarat Agro-Climatic Condition
}

\author{
Khushbu Kumari Gour, A.M. Patel, M.K. Sheshama*, K.G. Vyas and Girraj Sharma \\ Department of Agronomy, Chimanbhai Patel College of Agriculture, Sardarkrushinagar \\ Dantiwada Agricultural University, Sardarkrushinagar - 385 506, Gujarat, India \\ *Corresponding author
}

\author{
A B S T R A C T
}

\begin{tabular}{|l|}
\hline Ke y w or d s \\
Dill seed, INM, \\
Growth and yield \\
attributes. \\
\hline Article Info \\
\hline $\begin{array}{l}\text { Accepted: } \\
\text { 19 October } 2017 \\
\text { Available Online: } \\
10 \text { December } 2017\end{array}$ \\
\hline \hline
\end{tabular}

An experiment was conducted on loamy sand soil of Instructional Farm, Department of Agronomy, Chimanbhai Patel College of Agriculture, S.D. Agricultural University, Sardarkrushinagar (Gujarat) during the Rabi season of years 2011-12 and 2013-14 on to study "Integrated Nitrogen Management in Dill Seed (Anethum graveolens L.) under North Gujarat Agro-Climatic Condition" in Randomized Block Design (RBD) with three replications and Fourteen treatment combinations comprising urea, FYM, Vermicompost and bio-fertilizer application and Gujarat dill seed 3 was used as test crop. The pooled results of all the growth and yield attributes of dill seed such as plant height, number of branches per plant, number of umbel per plant, number of umbellate per umbel, number of seeds per umbellate and 1000- seed weight were significantly influenced by integrated nitrogen management. Significantly higher values of all the above parameters were recorded with $75 \%$ RDN through urea $+25 \%$ RDN through vermicompost $\left(\mathrm{T}_{6}\right)$ which ultimately reflected in higher seed $(1651 \mathrm{~kg} / \mathrm{ha})$ and stover $(2508 \mathrm{~kg} / \mathrm{ha})$ yields.

\section{Introduction}

Dill (Anethum graveolens L.) commonly known as Sowa in India is an annual, glabrous and aromatic herb belonging to the family Umbelliferae (Apiaceae). The dill fruit is a schizocarp with paired carpel that split apart at maturity to release two mericarps, commonly referred as "seed" (Callan et al., 2007). Spices need no introduction since they have been mentioned in the Vedas and the Bible and the fame of Indian spices is older than the recorded history. Spices are to be integral part of our Indian culture and also India is the world's largest producer, consumer and exporter of spices, that's why India is known as "Seed spice bowl". There are about 63 cultivated spices but only dozen of them are fully important for commercial or large scale cultivation. Among them cumin, dill seed and fennel etc. are vital Rabi seed spices, particular for arid and semi-arid regions of the country. Dill seed (Anethum graveolens L.) is one of the important medicinal and aromatic crops, belongs to family Umbelliferae. Dill fruits are also popularly used as carminative, aromatic stimulant and diuretic in Ayurvedic and Unani medicines. The Indian dill seed are known to contain more dillapiole $(36.0 \%)$ and less carvone $(19.5 \%)$ whereas European dill has more carvone $(45.9 \%)$ and less dillapiole 
(7\%) (Malhotra and Vashishtha, 2007). In India, Gujarat and Madhya Pradesh are the leading states for dill cultivation and production. Irrigated Rabi dill crop can be sown in the month of October. This is mainly because of lack of improved technology in the production of dill in India. Nutrient supply plays an important role in dill production. Under intensive cultivation, indiscriminate use of nitrogen and phosphorus fertilizers alone over long period could result in deficiency of nutrients other than that applied (Singh et al., 1999). Farmyard manure improves the soil properties and finally crop yields (Bhatia and Shukla, 1982). Results generated from series of long -term fertilizer experiments on other cropping systems have revealed that continuous use of high analysis chemical fertilizers increased the crop yield in initial years and adversely affect the sustainability at the later stage (Virmani, 1994). The crop response to inoculation with biofertilizers is known to vary with crop, cultivar, location, seasons, bacterial strain, level of soil fertility and interaction with native soil micro flora (Wani, 1990). At present very limited information is available in the literature on the effect of integrated nutrient management using chemical fertilizers, organic manures and biofertilizers on yield of dill in India. Field study was therefore conducted to study the effect of farmyard manure, biofertlizers and inorganic fertilizers on yield and nutrients uptake of European dill.

\section{Materials and Methods}

A field experiment was conducted at Agronomy Instructional Farm, Chimanbhai Patel College of Agriculture, Sardarkrushinagar Dantiwada Agricultural University, Sardarkrushinagar during Rabi season of two consecutive years 2011-12 and 2013-14 to study the "Integrated nitrogen management in Dill seed (Anethum graveolens L.) under North Gujarat AgroClimatic condition". Geographically, Sardarkrushinagar is situated at $24^{\circ}-19^{\prime}$ North latitude and $72^{\circ}-19^{\prime}$ East longitude with an elevation of 154.52 meter above the mean sea level. The soil of the experiment site was a sandy loam with $\mathrm{pH} 7.5$, electrical conductivity $0.15 \mathrm{dSm}$ at $25^{\circ} \mathrm{C}$, organic carbon $0.18 \%$, low in nitrogen, medium in phosphorus and potash. The experiment consisting of 14 treatments was laid out in a RBD with three replications with Gujarat dill seed 3 (variety) was used as test crop. These treatments are combinations comprising urea, FYM, vermicompost and bio-fertilizer application viz., $\mathrm{T}_{1}(100 \%$ RDN through urea), $\mathrm{T}_{2}$ (75 \% RDN through urea + Azospirillum, $\mathrm{T}_{3}(75 \% \mathrm{RDN}$ through urea + Azotobacter), $\mathrm{T}_{4}$ (75\% RDN through urea + Azospirillum + Azotobacter), $\mathrm{T}_{5}(75 \% \mathrm{RDN}$ through urea $+25 \%$ RDN through FYM), $\mathrm{T}_{6}$ (75\% RDN through urea $+25 \%$ RDN through vermicompost), $\mathrm{T}_{7} \quad(50 \%$ RDN through urea $+50 \%$ RDN through FYM), $\mathrm{T}_{8}$ (50\% RDN through urea $+50 \%$ RDN through vermicompost), $\mathrm{T}_{9} \quad(25 \%$ RDN through urea $+75 \%$ RDN through FYM), $\mathrm{T}_{10}$ (25\% RDN through urea $+75 \%$ RDN through vermicompost), $\mathrm{T}_{11}(100 \% \mathrm{RDN}$ through FYM), $\mathrm{T}_{12}$ (100\% RDN through vermicompost), $\mathrm{T}_{13} \quad(50 \% \mathrm{RDN}$ through $\mathrm{FYM}+50 \%$ RDN through vermicompost) and $\mathrm{T}_{14}(50 \%$ RDN through urea $+25 \%$ RDN through FYM + $25 \%$ RDN through vermicompost).

\section{Results and Discussion}

\section{Effect of INM on plant height}

Plant height At 30 DAS, treatment $\mathrm{T}_{1}$ being at par with $\mathrm{T}_{6}$ and $\mathrm{T}_{8}$ recorded significantly higher plant height $(15.5 \mathrm{~cm})$ during both the year and on pooled basis (Table 3 ). Among the treatments, application of $\mathrm{T}_{6}$ recorded significantly higher plant height of $(48.93 \mathrm{~cm})$ 
at 60 DAS which was statically at par with treatments $T_{1}, T_{3}, T_{4}, T_{8}$ and $T_{5}$ during second year (Table 1). According to pooled data basis significantly higher plant height $(48.3 \mathrm{~cm})$ was observed in $\mathrm{T}_{6}$ treatment which was at par with treatment $\mathrm{T}_{8}$ and $\mathrm{T}_{5}$ and similar trend was observed in first year.

At 90 DAS, treatment $T_{6}$ showed significantly higher plant height $(102.0 \mathrm{~cm})$ which was at par with $\mathrm{T}_{1}, \mathrm{~T}_{4}, \mathrm{~T}_{5}, \mathrm{~T}_{8}, \mathrm{~T}_{9}, \mathrm{~T}_{10}, \mathrm{~T}_{13}$ and $\mathrm{T}_{14}$ during first year and second year again treatment $\mathrm{T}_{6}$ found significantly higher plant height and which was at par with $\mathrm{T}_{4}, \mathrm{~T}_{5}, \mathrm{~T}_{8}$, $\mathrm{T}_{9}$ and $\mathrm{T}_{10}$. According to pooled data significantly higher plant height $(102.98 \mathrm{~cm})$ was observed in $T_{6}$ which was at par with $T_{5}$ and $\mathrm{T}_{6}$ treatment (Table 1). During first year significantly higher plant height $(111.0 \mathrm{~cm})$ was recorded in $\mathrm{T}_{6}$ which was at par with $\mathrm{T}_{1}$, $\mathrm{T}_{4}, \mathrm{~T}_{5}$ and $\mathrm{T}_{8}$ at 120 DAS. Similar trend was observed in second year. According to mean data of both years, significantly higher plant height was recorded in $\mathrm{T}_{6}$ which was at par with $\mathrm{T}_{5}$ and $\mathrm{T}_{8}$ treatment at 120 DAS (Table 2).

At maturity, treatment $\mathrm{T}_{6}$ observed significantly higher plant height $(110 \mathrm{~cm})$ which was at par with $\mathrm{T}_{1}, \mathrm{~T}_{4}, \mathrm{~T}_{5}, \mathrm{~T}_{7}, \mathrm{~T}_{8}, \mathrm{~T}_{9}$ and $\mathrm{T}_{10}$ during first year and second year again treatment $T_{6}$ found significantly higher plant height and which was at par with $\mathrm{T}_{1}$ and $\mathrm{T}_{8}$. According to pooled data the highest plant height $(111.06 \mathrm{~cm})$ was recorded in $\mathrm{T}_{6}$ treatment (Table 2).

This might be due an adequate amount of nitrogen supply which enhanced the cell division and cell enlargement and helped to convert more solar energy in to chemical energy. Application of inorganic with organic manure might have supplied the adequate and continuous amount of nutrient at different stages due to release of sufficient amount of nutrient by mineralization at a constant level that resulted in higher plant growth. The results are conformity with those reported by Selvarajan and Chezhiyan, (2001), Derzi et al., (2005) and Hellal et al., (2011).

\section{Number of branches per plant}

Significantly higher number of branches per plant (17.08, branches) was recorded with $\left(\mathrm{T}_{6}\right)$, which was being at par with $\left(\mathrm{T}_{8}\right)$ and $\left(\mathrm{T}_{5}\right)$ on pooled basis (Table 4). Similar trend was observed in both the year.

More availability of nutrients under organic and inorganic fertilizer or integrated nitrogen management system might have enhanced meristematic activities and size of cells as well as formation and functioning of protoplasm which consequently improved the crop growth in terms of number of branches per plant also reported by Kumar et al., (2002)

\section{Days to $50 \%$ flowering}

Data on days to 50 per cent flowering of dill seed as influenced by different treatment of integrated nitrogen management during both the year as well as pooled basis are presented in Table 5. The result revealed that integrated nitrogen management treatments did not exert any significant influence on days to 50 per cent flowering during both the years as well as pooled analysis basis.

\section{Number of days to maturity}

Data on days to maturity of dill seed as influenced by different treatment of integrated nitrogen management during both the year as well as pooled basis are presented in Table 6 . The result found that integrated nitrogen management treatments did not show any significant influence on days to maturity during both the year as well as in pooled analysis. 
Table.1 Effect of integrated nitrogen management on plant height $(\mathrm{cm})$ at 60 and 90 DAS of dill seed

\begin{tabular}{|c|c|c|c|c|c|c|}
\hline \multirow[t]{3}{*}{ TREATMENTS } & \multicolumn{6}{|c|}{ Plant height (cm) } \\
\hline & \multicolumn{3}{|c|}{60 DAS } & \multicolumn{3}{|c|}{90 DAS } \\
\hline & 2011-12 & 2013-14 & Pooled & 2011-12 & $\begin{array}{c}2013- \\
14\end{array}$ & Pooled \\
\hline $\mathbf{T}_{1}: 100 \%$ RDN through urea & 41.62 & 42.00 & 41.81 & 91.57 & 92.17 & 91.87 \\
\hline $\mathbf{T}_{2}: 75 \%$ RDN through urea + Azospirillum & 35.67 & 39.00 & 37.33 & 84.97 & 85.00 & 84.98 \\
\hline $\mathbf{T}_{5}: 75 \%$ RDN through urea $+25 \%$ RDN through FYM & 43.50 & 45.83 & 44.67 & 97.63 & 97.83 & 97.73 \\
\hline $\mathbf{T}_{6}: 75 \%$ RDN through urea $+25 \%$ RDN through Vermicompost & 47.70 & 48.93 & 48.32 & 101.97 & 104.00 & 102.98 \\
\hline $\mathbf{T}_{7}: 50 \%$ RDN through urea $+50 \%$ RDN through FYM & 40.87 & 41.17 & 41.02 & 80.33 & 89.33 & 84.83 \\
\hline $\mathbf{T}_{11}: 100 \%$ RDN through FYM & 35.27 & 35.33 & 35.30 & 82.87 & 83.33 & 83.10 \\
\hline $\mathbf{T}_{\mathbf{1 2}}: 100 \%$ RDN through Vermicompost & 36.80 & 36.93 & 36.87 & 80.87 & 85.53 & 83.20 \\
\hline $\begin{array}{l}\mathbf{T}_{\mathbf{1 3}}: 50 \% \text { RDN through FYM }+50 \% \text { RDN through } \\
\text { Vermicompost }\end{array}$ & 37.32 & 40.98 & 39.15 & 89.53 & 89.67 & 89.60 \\
\hline $\begin{array}{l}\mathbf{T}_{14}: 50 \% \text { RDN through urea }+25 \% \text { RDN through FYM }+25 \% \\
\text { RDN through Vermicompost }\end{array}$ & 40.30 & 41.70 & 41.00 & 91.23 & 92.83 & 92.03 \\
\hline S.Em. \pm & 1.932 & 2.400 & 1.334 & 4.372 & 3.487 & 2.421 \\
\hline C.D. $(\mathrm{P}=0.05)$ & 5.617 & 6.977 & 3.786 & 12.708 & 10.136 & 6.871 \\
\hline C.V.\% & 8.28 & 9.91 & 9.16 & 8.38 & 6.55 & 7.50 \\
\hline
\end{tabular}


Table.2 Effect of integrated nitrogen management on plant height $(\mathrm{cm})$ at 120 DAS and at maturity of dill seed

\begin{tabular}{|c|c|c|c|c|c|c|}
\hline \multirow[t]{3}{*}{ TREATMENTS } & \multicolumn{6}{|c|}{ Plant height (cm) } \\
\hline & \multicolumn{3}{|c|}{120 DAS } & \multicolumn{3}{|c|}{ At maturity } \\
\hline & $2011-12$ & 2013-14 & Pooled & $2011-12$ & $\begin{array}{c}2013- \\
14\end{array}$ & Pooled \\
\hline $\mathbf{T}_{\mathbf{1}}: 100 \% \mathrm{RDN}$ through urea & 104.00 & 104.73 & 104.37 & 105.67 & 106.13 & 105.90 \\
\hline $\mathbf{T}_{\mathbf{2}}: 75 \%$ RDN through urea + Azospirillum & 94.33 & 97.67 & 96.00 & 97.00 & 100.33 & 98.67 \\
\hline $\mathbf{T}_{5}: 75 \%$ RDN through urea $+25 \%$ RDN through FYM & 104.73 & 105.00 & 104.87 & 104.00 & 105.00 & 104.50 \\
\hline $\mathbf{T}_{6}: 75 \%$ RDN through urea $+25 \%$ RDN through Vermicompost & 110.00 & 111.19 & 110.60 & 110.15 & 111.97 & 111.06 \\
\hline $\mathbf{T}_{7}: 50 \%$ RDN through urea $+50 \%$ RDN through FYM & 99.33 & 100.00 & 99.67 & 103.00 & 104.00 & 103.50 \\
\hline $\mathbf{T}_{8}: 50 \%$ RDN through urea $+50 \%$ RDN through Vermicompost & 104.33 & 105.67 & 105.00 & 106.03 & 107.73 & 106.88 \\
\hline $\mathbf{T}_{\mathbf{1 1}}: 100 \% \mathrm{RDN}$ through FYM & 92.67 & 94.33 & 93.50 & 98.86 & 97.67 & 98.27 \\
\hline $\mathbf{T}_{\mathbf{1 2}}: 100 \%$ RDN through Vermicompost & 94.67 & 96.87 & 95.77 & 98.82 & 100.00 & 99.41 \\
\hline $\begin{array}{l}\mathbf{T}_{\mathbf{1 3}}: 50 \% \text { RDN through FYM + } 50 \% \text { RDN through } \\
\text { Vermicompost }\end{array}$ & 93.23 & 94.67 & 93.95 & 97.85 & 100.33 & 99.09 \\
\hline $\begin{array}{l}\text { T14: }_{14} 50 \% \text { RDN through urea }+25 \% \text { RDN through FYM }+25 \% \\
\text { RDN through Vermicompost }\end{array}$ & 93.67 & 94.73 & 94.20 & 99.67 & 99.67 & 99.67 \\
\hline S.Em. \pm & 3.170 & 3.343 & 1.995 & 2.545 & 2.206 & 1.458 \\
\hline C.D. $(\mathrm{P}=0.05)$ & 9.215 & 9.717 & 5.661 & 7.397 & 6.413 & 4.138 \\
\hline C.V.\% & 5.53 & 5.76 & 5.65 & 4.32 & 3.70 & 4.02 \\
\hline
\end{tabular}


Table.3 Effect of integrated nitrogen management on plant height $(\mathrm{cm})$ at 30 DAS of dill seed

\begin{tabular}{|l|c|c|c|}
\hline \multicolumn{1}{|c|}{ TREATMENTS } & \multicolumn{3}{c|}{ Plant height (cm) at 30 DAS } \\
\cline { 2 - 4 } & $\mathbf{2 0 1 1 - 1 2}$ & $\mathbf{2 0 1 3 - 1 4}$ & Pooled \\
\hline $\mathbf{T}_{\mathbf{1}}: 100 \%$ RDN through urea & $\mathbf{1 5 . 3 3}$ & $\mathbf{1 5 . 6 7}$ & $\mathbf{1 5 . 5 0}$ \\
\hline $\mathbf{T}_{\mathbf{2}}: 75 \%$ RDN through urea + Azospirillum & 9.83 & 10.13 & 9.98 \\
\hline $\mathbf{T}_{\mathbf{3}}: 75 \%$ RDN through urea + Azotobacter & 9.63 & 9.73 & 9.68 \\
\hline $\mathbf{T}_{\mathbf{4}}: 75 \%$ RDN through urea + Azospirillum + Azotobacter & 10.90 & 11.17 & 11.03 \\
\hline $\mathbf{T}_{\mathbf{5}}: 75 \%$ RDN through urea + 25 \% RDN through FYM & 11.58 & 11.67 & 11.63 \\
\hline $\mathbf{T}_{\mathbf{6}}: 75 \%$ RDN through urea + 25 \% RDN through vermicompost & 13.77 & 14.80 & 14.28 \\
\hline $\mathbf{T}_{\mathbf{7}}: 50 \%$ RDN through urea + 50 \% RDN through FYM & 11.47 & 11.75 & 11.61 \\
\hline $\mathbf{T}_{\mathbf{8}}: 50 \%$ RDN through urea + 50 \% RDN through vermicompost & 13.37 & 13.57 & 13.47 \\
\hline $\mathbf{T}_{\mathbf{9}}: 25 \%$ RDN through urea + 75\% RDN through FYM & 11.23 & 11.53 & 11.38 \\
\hline $\mathbf{T}_{\mathbf{1 0}}: 25 \%$ RDN through urea + 75\% RDN through vermicompost & 11.33 & 11.50 & 11.42 \\
\hline $\mathbf{T}_{\mathbf{1 1}}: 100 \%$ RDN through FYM & 9.83 & 10.20 & 10.02 \\
\hline $\mathbf{T}_{\mathbf{1 2}}: 100 \%$ RDN through vermicompost & 10.30 & 10.40 & 10.35 \\
\hline $\mathbf{T}_{\mathbf{1 3}}: 50 \%$ RDN through FYM + 50 \% RDN through vermicompost & 11.50 & 11.67 & 11.58 \\
\hline $\mathbf{T}_{\mathbf{1 4}}: 50 \%$ RDN through urea + 25\% RDN through FYM + 25 \% & 11.73 & 11.83 & 11.78 \\
RDN through vermicompost & & & \\
\hline S.Em. \pm & 1.007 & 1.183 & 0.673 \\
\hline C.D. (P=0.05) & 2.927 & 3.440 & 1.909 \\
\hline C.V.\% & 15.09 & 17.32 & 16.27 \\
\hline
\end{tabular}

Table.4 Effect of integrated nitrogen management on number of branches per plant of dill seed

\section{TREATMENTS}

$\mathbf{T}_{\mathbf{1}}: 100 \%$ RDN through urea

$\mathbf{T}_{\mathbf{2}}: 75 \%$ RDN through urea + Azospirillum

$\mathbf{T}_{\mathbf{3}}: 75 \%$ RDN through urea + Azotobacter

$\mathbf{T}_{\mathbf{4}}: 75 \%$ RDN through urea + Azospirillum + Azotobacter

$\mathbf{T}_{5}: 75 \%$ RDN through urea $+25 \%$ RDN through FYM

$\mathbf{T}_{\mathbf{6}}: 75 \%$ RDN through urea $+25 \%$ RDN through Vermicompost

$\mathbf{T}_{7}: 50 \%$ RDN through urea $+50 \%$ RDN through FYM

$\mathbf{T}_{\mathbf{8}}: 50 \%$ RDN through urea $+50 \%$ RDN through Vermicompost

$\mathbf{T}_{\mathbf{9}}: 25 \%$ RDN through urea $+75 \%$ RDN through FYM

$\mathbf{T}_{\mathbf{1 0}}: 25 \%$ RDN through urea $+75 \%$ RDN through Vermicompost

$\mathbf{T}_{\mathbf{1 1}}: 100 \%$ RDN through FYM

$\mathbf{T}_{\mathbf{1 2}}: 100 \%$ RDN through Vermicompost

$\mathbf{T}_{13}: 50 \%$ RDN through FYM + 50\% RDN through Vermicompost

$\mathbf{T}_{14}: 50 \%$ RDN through urea $+25 \%$ RDN through FYM + $25 \%$

RDN through Vermicompost

\begin{tabular}{|c|c|c|c|}
\hline S.Em. \pm & 0.710 & 0.809 & 0.466 \\
\hline C.D. $(\mathrm{P}=0.05)$ & 2.065 & 2.353 & 1.323 \\
\hline C.V.\% & 8.78 & 9.80 & 9.32 \\
\hline
\end{tabular}


Table.5 Effect of integrated nitrogen management on Days to $50 \%$ flowering of dill seed

\begin{tabular}{|c|c|c|c|}
\hline \multirow[t]{2}{*}{ TREATMENTS } & \multicolumn{3}{|c|}{ Days to $50 \%$ flowering } \\
\hline & 2011-12 & 2013-14 & Pooled \\
\hline $\mathbf{T}_{\mathbf{1}}: 100 \%$ RDN through urea & 78 & 79 & 79 \\
\hline $\mathbf{T}_{2}: 75 \%$ RDN through urea + Azospirillum & 79 & 79 & 79 \\
\hline $\mathbf{T}_{3}: 75 \%$ RDN through urea + Azotobacter & 80 & 81 & 81 \\
\hline $\mathbf{T}_{4}: 75 \%$ RDN through urea + Azospirillum + Azotobacter & 76 & 78 & 77 \\
\hline $\mathbf{T}_{5}: 75 \%$ RDN through urea $+25 \%$ RDN through FYM & 87 & 87 & 87 \\
\hline $\mathbf{T}_{6}: 75 \%$ RDN through urea $+25 \%$ RDN through Vermicompost & 84 & 85 & 85 \\
\hline $\mathbf{T}_{7}: 50 \%$ RDN through urea $+50 \%$ RDN through FYM & 79 & 80 & 80 \\
\hline $\mathbf{T}_{8}: 50 \%$ RDN through urea $+50 \%$ RDN through Vermicompost & 86 & 87 & 86 \\
\hline $\mathbf{T}_{9}: 25 \%$ RDN through urea $+75 \%$ RDN through FYM & 86 & 86 & 86 \\
\hline $\mathbf{T}_{\mathbf{1 0}}: 25 \%$ RDN through urea $+75 \%$ RDN through Vermicompost & 82 & 83 & 83 \\
\hline $\mathbf{T}_{11}: 100 \%$ RDN through FYM & 83 & 83 & 83 \\
\hline $\mathbf{T}_{\mathbf{1 2}}: 100 \%$ RDN through Vermicompost & 81 & 82 & 81 \\
\hline $\mathbf{T}_{13}: 50 \%$ RDN through FYM + $50 \%$ RDN through Vermicompost & 81 & 81 & 81 \\
\hline $\begin{array}{l}\mathbf{T}_{14}: 50 \% \text { RDN through urea }+25 \% \text { RDN through FYM }+25 \% \\
\text { RDN through Vermicompost }\end{array}$ & 80 & 80 & 80 \\
\hline S.Em. \pm & 2.99 & 2.97 & 1.83 \\
\hline C.D. $(\mathrm{P}=0.05)$ & NS & NS & NS \\
\hline C.V.\% & 6.34 & 6.27 & 6.30 \\
\hline
\end{tabular}

Table.6 Effect of integrated nitrogen management on Days to maturity of dill seed

\begin{tabular}{|c|c|c|c|}
\hline \multirow[t]{2}{*}{ TREATMENTS } & \multicolumn{3}{|c|}{ Days to maturity } \\
\hline & 2011-12 & 2013-14 & Pooled \\
\hline $\mathbf{T}_{\mathbf{1}}: 100 \%$ RDN through urea & 122 & 124 & 123 \\
\hline $\mathbf{T}_{\mathbf{2}}: 75 \%$ RDN through urea + Azospirillum & 123 & 123 & 123 \\
\hline $\mathbf{T}_{3}: 75 \%$ RDN through urea + Azotobacter & 125 & 125 & 125 \\
\hline $\mathbf{T}_{\mathbf{4}}: 75 \%$ RDN through urea + Azospirillum + Azotobacter & 122 & 122 & 122 \\
\hline $\mathbf{T}_{5}: 75 \%$ RDN through urea $+25 \%$ RDN through FYM & 136 & 137 & 137 \\
\hline $\mathbf{T}_{6}: 75 \%$ RDN through urea $+25 \%$ RDN through Vermicompost & 135 & 136 & 135 \\
\hline $\mathbf{T}_{7}: 50 \%$ RDN through urea $+50 \%$ RDN through FYM & 124 & 125 & 125 \\
\hline $\mathbf{T}_{8}: 50 \%$ RDN through urea $+50 \%$ RDN through Vermicompost & 135 & 135 & 135 \\
\hline $\mathbf{T}_{\mathbf{9}}: 25 \%$ RDN through urea $+75 \%$ RDN through FYM & 134 & 134 & 134 \\
\hline $\mathbf{T}_{10}: 25 \%$ RDN through urea $+75 \%$ RDN through Vermicompost & 128 & 129 & 129 \\
\hline $\mathbf{T}_{\mathbf{1 1}}: 100 \%$ RDN through FYM & 130 & 130 & 130 \\
\hline $\mathbf{T}_{\mathbf{1 2}}: 100 \%$ RDN through Vermicompost & 126 & 128 & 127 \\
\hline $\mathbf{T}_{13}: 50 \%$ RDN through FYM + 50\% RDN through Vermicompost & 126 & 127 & 127 \\
\hline $\begin{array}{l}\mathbf{T}_{14}: 50 \% \text { RDN through urea }+25 \% \text { RDN through FYM }+25 \% \\
\text { RDN through Vermicompost }\end{array}$ & 124 & 125 & 124 \\
\hline S.Em. \pm & 3.72 & 3.49 & 2.21 \\
\hline C.D. $(\bar{P}=0.05)$ & NS & NS & $\mathrm{NS}$ \\
\hline C.V.\% & 5.03 & 4.70 & 4.87 \\
\hline
\end{tabular}


Table.7 Effect of integrated nitrogen management on number of umbels per plant (Effective and Non-effective) of dill seed

\begin{tabular}{|c|c|c|c|c|c|c|}
\hline \multirow[t]{2}{*}{ TREATMENTS } & \multicolumn{3}{|c|}{$\begin{array}{l}\text { Number of umbels per } \\
\text { plant (Effective) }\end{array}$} & \multicolumn{3}{|c|}{$\begin{array}{l}\text { Number of umbels per } \\
\text { plant (Non-effective) }\end{array}$} \\
\hline & 2011- & 2013- & $\begin{array}{c}\text { Poole } \\
\text { d }\end{array}$ & 12 & 14 & $\begin{array}{c}\text { Poole } \\
\text { d }\end{array}$ \\
\hline $\mathbf{T}_{1}: 100 \%$ RDN through urea & 11.13 & 11.20 & 11.17 & 4.80 & 4.93 & 4.87 \\
\hline $\mathbf{T}_{2}: 75 \%$ RDN through urea + Azospirillum & 9.80 & 10.20 & 10.00 & 4.67 & 4.77 & 4.72 \\
\hline $\mathbf{T}_{3}: 75 \%$ RDN through urea + Azotobacter & 9.73 & 10.43 & 10.08 & 3.87 & 3.99 & 3.93 \\
\hline $\mathbf{T}_{4}: 75 \%$ RDN through urea + Azospirillum + Azotobacter & 11.33 & 12.10 & 11.72 & 4.03 & 4.27 & 4.15 \\
\hline $\mathbf{T}_{5}: 75 \%$ RDN through urea $+25 \%$ RDN through FYM & 12.63 & 13.23 & 12.93 & 5.27 & 5.67 & 5.47 \\
\hline $\mathbf{T}_{6}: 75 \%$ RDN through urea $+25 \%$ RDN through vermicompost & 13.88 & 14.20 & 14.04 & 5.44 & 5.68 & 5.56 \\
\hline $\mathbf{T}_{7}: 50 \%$ RDN through urea $+50 \%$ RDN through FYM & 10.33 & 10.53 & 10.43 & 4.20 & 4.40 & 4.30 \\
\hline $\mathbf{T}_{8}: 50 \%$ RDN through urea $+50 \%$ RDN through vermicompost & 13.00 & 13.20 & 13.10 & 4.87 & 5.13 & 5.00 \\
\hline $\mathbf{T}_{9}: 25 \%$ RDN through urea $+75 \%$ RDN through FYM & 9.67 & 10.13 & 9.90 & 4.43 & 4.50 & 4.47 \\
\hline $\mathbf{T}_{10}: 25 \%$ RDN through urea+75\% RDN through vermicompost & 9.85 & 10.40 & 10.13 & 5.10 & 5.23 & 5.17 \\
\hline $\mathbf{T}_{11}: 100 \%$ RDN through FYM & 9.03 & 9.57 & 9.30 & 5.23 & 5.37 & 5.30 \\
\hline $\mathbf{T}_{\mathbf{1 2}}: 100 \%$ RDN through vermicompost & 10.47 & 10.90 & 10.68 & 3.67 & 3.78 & 3.73 \\
\hline $\mathbf{T}_{\mathbf{1 3}}: 50 \%$ RDN through FYM $+50 \%$ RDN through vermicompost & 10.67 & 10.80 & 10.73 & 3.91 & 4.00 & 3.95 \\
\hline $\begin{array}{l}\mathbf{T}_{\mathbf{1 4}}: 50 \% \text { RDN through urea }+25 \% \mathrm{RDN} \text { through FYM }+25 \% \\
\text { RDN through vermicompost }\end{array}$ & 10.77 & 11.23 & 11.00 & 4.10 & 4.17 & 4.13 \\
\hline S.Em. \pm & 0.620 & 0.685 & 0.400 & 0.211 & 0.228 & 0.135 \\
\hline C.D. $(\mathrm{P}=0.05)$ & 1.802 & 1.990 & 1.135 & 0.614 & 0.664 & 0.382 \\
\hline C.V.\% & 9.87 & 10.50 & 10.20 & 8.05 & 8.41 & 8.24 \\
\hline
\end{tabular}

Table.8 Effect of integrated nitrogen management on number of umbellets per umbel of dill seed

\begin{tabular}{|c|c|c|c|}
\hline \multirow[t]{2}{*}{ TREATMENTS } & \multicolumn{3}{|c|}{ Number of umbellets per umbel } \\
\hline & 2011-12 & 2013-14 & Pooled \\
\hline $\mathbf{T}_{1}: 100 \%$ RDN through urea & 16.30 & 16.67 & 16.48 \\
\hline $\mathbf{T}_{2}: 75 \%$ RDN through urea + Azospirillum & 14.51 & 15.21 & 14.86 \\
\hline $\mathbf{T}_{3}: 75 \%$ RDN through urea + Azotobacter & 16.17 & 16.39 & 16.28 \\
\hline $\mathbf{T}_{4}: 75 \%$ RDN through urea + Azospirillum + Azotobacter & 17.57 & 17.73 & 17.65 \\
\hline $\mathbf{T}_{5}: 75 \%$ RDN through urea $+25 \%$ RDN through FYM & 17.60 & 18.24 & 17.92 \\
\hline $\mathbf{T}_{6}: 75 \%$ RDN through urea $+25 \%$ RDN through Vermicompost & 18.56 & 19.97 & 19.27 \\
\hline $\mathbf{T}_{7}: 50 \%$ RDN through urea $+50 \%$ RDN through FYM & 16.17 & 16.73 & 16.45 \\
\hline $\mathbf{T}_{8}: 50 \%$ RDN through urea $+50 \%$ RDN through Vermicompost & 17.91 & 18.20 & 18.06 \\
\hline $\mathbf{T}_{\mathbf{9}}: 25 \%$ RDN through urea $+75 \%$ RDN through FYM & 15.80 & 16.88 & 16.34 \\
\hline $\mathbf{T}_{10}: 25 \%$ RDN through urea $+75 \%$ RDN through Vermicompost & 16.13 & 16.37 & 16.25 \\
\hline $\mathbf{T}_{11}: 100 \%$ RDN through FYM & 13.59 & 14.40 & 13.99 \\
\hline $\mathbf{T}_{\mathbf{1 2}}: 100 \%$ RDN through Vermicompost & 14.10 & 14.97 & 14.53 \\
\hline $\mathbf{T}_{\mathbf{1 3}}: 50 \%$ RDN through FYM + 50\% RDN through Vermicompost & 15.51 & 16.49 & 16.00 \\
\hline $\begin{array}{l}\mathbf{T}_{14}: 50 \% \text { RDN through urea }+25 \% \text { RDN through FYM }+25 \% \text { RDN } \\
\text { through Vermicompost }\end{array}$ & 16.20 & 16.57 & 16.38 \\
\hline S.Em. \pm & 0.782 & 0.881 & 0.510 \\
\hline C.D. $(\mathrm{P}=0.05)$ & 2.274 & 2.560 & 1.448 \\
\hline C.V.\% & 8.39 & 9.10 & 8.76 \\
\hline
\end{tabular}


Table.9 Effect of integrated nitrogen management on number of seeds per umbellets of dill seed

\begin{tabular}{|l|c|c|c|}
\hline \multicolumn{1}{|c|}{ TREATMENTS } & \multicolumn{3}{c|}{ Number of seeds per umbellets } \\
\cline { 2 - 4 } & $\mathbf{2 0 1 1 - 1 2}$ & $\mathbf{2 0 1 3 - 1 4}$ & Pooled \\
\hline $\mathbf{T}_{\mathbf{1}}: 100 \%$ RDN through urea & 12.63 & 13.03 & 12.83 \\
\hline $\mathbf{T}_{\mathbf{2}}: 75 \%$ RDN through urea + Azospirillum & 10.93 & 11.87 & 11.40 \\
\hline $\mathbf{T}_{\mathbf{3}}: 75 \%$ RDN through urea + Azotobacter & 11.85 & 12.05 & 11.95 \\
\hline $\mathbf{T}_{\mathbf{4}}: 75 \%$ RDN through urea + Azospirillum + Azotobacter & 12.92 & 13.40 & 13.16 \\
\hline $\mathbf{T}_{\mathbf{5}}: 75 \%$ RDN through urea + 25\% RDN through FYM & 13.00 & 13.50 & 13.25 \\
\hline $\mathbf{T}_{\mathbf{6}}: 75 \%$ RDN through urea + 25\% RDN through Vermicompost & $\mathbf{1 3 . 7 3}$ & $\mathbf{1 4 . 5 7}$ & $\mathbf{1 4 . 1 5}$ \\
\hline $\mathbf{T}_{\mathbf{7}}: 50 \%$ RDN through urea + 50\% RDN through FYM & 11.77 & 11.99 & 11.88 \\
\hline $\mathbf{T}_{\mathbf{8}}: 50 \%$ RDN through urea + 50\% RDN through Vermicompost & 13.33 & 13.63 & 13.48 \\
\hline $\mathbf{T}_{\mathbf{9}}: 25 \%$ RDN through urea + 75\% RDN through FYM & 10.80 & 11.27 & 11.03 \\
\hline $\mathbf{T}_{\mathbf{1 0}}: 25 \%$ RDN through urea + 75\% RDN through Vermicompost & 11.16 & 11.37 & 11.26 \\
\hline $\mathbf{T}_{\mathbf{1 1}}: 100 \%$ RDN through FYM & 10.70 & 11.07 & 10.88 \\
\hline $\mathbf{T}_{\mathbf{1 2}}: 100 \%$ RDN through Vermicompost & 11.58 & 11.73 & 11.66 \\
\hline $\mathbf{T}_{\mathbf{1 3}}: 50 \%$ RDN through FYM + 50\% RDN through Vermicompost & 12.37 & 12.45 & 12.41 \\
\hline $\mathbf{T}_{\mathbf{1 4}}: 50 \%$ RDN through urea + 25\% RDN through FYM + 25 \% & 12.33 & 12.50 & 12.42 \\
\hline RDN through Vermicompost & & & 0.318 \\
\hline S.Em. \pm & 0.590 & 0.436 & 0.901 \\
\hline C.D. (P=0.05) & 1.715 & 1.267 & 0.32 \\
\hline
\end{tabular}

Table.10 Effect of integrated nitrogen management on 1000-seed weight of dill seed

\begin{tabular}{|l|c|c|c|}
\hline \multicolumn{1}{|c|}{ TREATMENTS } & \multicolumn{3}{c|}{ 1000-seed weight (g) } \\
\cline { 2 - 4 } & $\mathbf{2 0 1 1 - 1 2}$ & $\mathbf{2 0 1 3 - 1 4}$ & Pooled \\
\hline $\mathbf{T}_{\mathbf{1}}: 100 \%$ RDN through urea & 3.62 & 3.80 & 3.71 \\
\hline $\mathbf{T}_{\mathbf{2}}: 75 \%$ RDN through urea + Azospirillum & 3.34 & 3.37 & 3.35 \\
\hline $\mathbf{T}_{\mathbf{3}}: 75 \%$ RDN through urea + Azotobacter & 3.38 & 3.44 & 3.41 \\
\hline $\mathbf{T}_{\mathbf{4}}: 75 \%$ RDN through urea + Azospirillum + Azotobacter & 3.43 & 3.57 & 3.50 \\
\hline $\mathbf{T}_{\mathbf{5}}: 75 \%$ RDN through urea + 25 \% RDN through FYM & 3.83 & 4.02 & 3.93 \\
\hline $\mathbf{T}_{\mathbf{6}}: 75 \%$ RDN through urea + 25 \% RDN through vermicompost & $\mathbf{4 . 0 5}$ & $\mathbf{4 . 1 5}$ & $\mathbf{4 . 1 0}$ \\
\hline $\mathbf{T}_{\mathbf{7}}: 50 \%$ RDN through urea + 50 \% RDN through FYM & 3.35 & 3.37 & 3.36 \\
\hline $\mathbf{T}_{\mathbf{8}}: 50 \%$ RDN through urea + 50 \% RDN through vermicompost & 4.00 & 4.03 & 4.01 \\
\hline $\mathbf{T}_{\mathbf{9}}: 25 \%$ RDN through urea + 75\% RDN through FYM & 3.32 & 3.34 & 3.33 \\
\hline $\mathbf{T}_{\mathbf{1 0}}: 25 \%$ RDN through urea + 75\% RDN through vermicompost & 3.28 & 3.32 & 3.30 \\
\hline $\mathbf{T}_{\mathbf{1 1}}: 100 \%$ RDN through FYM & 3.23 & 3.28 & 3.25 \\
\hline $\mathbf{T}_{\mathbf{1 2}}: 100 \%$ RDN through vermicompost & 3.26 & 3.28 & 3.27 \\
\hline $\mathbf{T}_{\mathbf{1 3}}: 50 \%$ RDN through FYM + 50 \% RDN through vermicompost & 3.61 & 3.67 & 3.64 \\
\hline $\mathbf{T}_{\mathbf{1 4}}: 50 \%$ RDN through urea + 25\% RDN through FYM + 25 \% & 3.62 & 3.68 & 3.65 \\
RDN through vermicompost & & & \\
\hline S.Em. \pm & 0.140 & 0.115 & 0.079 \\
\hline C.D. (P=0.05) & 0.408 & 0.334 & 0.223 \\
\hline & 6.89 & 5.54 & 6.24 \\
\hline
\end{tabular}


Number of umbels per plant (Effective or non-Effective)

The data on number of effective umbels per plant as influenced by integrated nitrogen management treatments during both the year as well as pooled basis are given in Table 7 . During first year and second year significantly higher number of effective umbels per plant (13.88 and 14.20 umbels) was recorded with $\left(\mathrm{T}_{6}\right)$, which was being at par with $\left(\mathrm{T}_{8}\right)$ and $\left(\mathrm{T}_{5}\right)$. According to pooled data basis treatment $T_{6}$ recorded significantly higher number of effective umbels per plant (14.04, umbels) which was at par with treatment $T_{8}$ and $T_{5}$. Similar result observed during both the year.

The data pertaining to number of noneffective umbels per plant recorded at maturity of crop growth during both the years as well as pooled basis are presented in Table 1. According to pooled data basis treatment $\mathrm{T}_{11}$ recorded significantly higher number of non-effective umbels per plant (5.6, umbels) which was at par with treatment $T_{12}, T_{10}, T_{3}$. This result is might be owing to the beneficial effect of vermicompost and FYM influencing the growth and yield attributes favorably supported by findings of several researchers Meena et al., (2007) and Darzi et al., (2012).

\section{Number of umbellates per umbel}

The mean data on number of umbellates per umbel recorded at maturity as affected by different integrated nitrogen management treatment along with both the year as well as pooled basis statistical inferences are presented in Table 8. During the year 201112 , treatment $\left(T_{6}\right)$ recorded significantly higher number of umbellates per umbel (18.56, umbellates) which was being at par with $\left(\mathrm{T}_{1}\right),\left(\mathrm{T}_{4}\right),\left(\mathrm{T}_{8}\right)$ and $\left(\mathrm{T}_{5}\right)$. During second year (2013-14) again treatment $T_{6}$ recorded significantly higher number of umbellates per umbel (19.97, umbellates) which was being at par with treatment $\mathrm{T}_{4}, \mathrm{~T}_{5}$ and $\mathrm{T}_{8}$. According to pooled data basis treatment $T_{6}$, recorded significantly higher number of umbellates per umbel (19.27, umbellates), which was at par with treatment $\mathrm{T}_{8}$ and $\mathrm{T}_{5}$.

\section{Number of seeds per umbellates}

During first year significantly maximum number of seeds per umbellates (13.73 seeds) was recorded with $T_{6}$ which was at par with treatments $\mathrm{T}_{8}, \mathrm{~T}_{5}, \mathrm{~T}_{4}, \mathrm{~T}_{1}, \mathrm{~T}_{13}$ and $\mathrm{T}_{14}$. During the year 2013-14, significantly higher number of seeds per umbellates (14.57 seeds) was recorded with $\mathrm{T}_{6}$ which was at par with application of treatments $\mathrm{T}_{8}, \mathrm{~T}_{4}$ and $\mathrm{T}_{5}$ (Table 9). According to pooled data basis treatment $\mathrm{T}_{6}$ recorded significantly higher number of seeds per umbellates (14.15, seeds) which was at par with treatment $T_{8}$ and $T_{5}$. The increased photosynthesis might have enhanced number of flower and their fertilization resulting in number of flowers and their fertilization resulting in higher number of seeds per umbellate and agreed with the findings of several researchers by Meena et al., (2007) and Darzi et al., (2012).

\section{0 seed weight $(\mathrm{g})$}

Significantly higher 1000 seed weight $(4.10 \mathrm{~g})$ was recorded with $\left(\mathrm{T}_{6}\right)$, which was being at par with $\left(\mathrm{T}_{8}\right)$ and $\left(\mathrm{T}_{5}\right)$ during the both year and on pooled basis (Table 10).

\section{References}

Bhatia, K.S. and Shukla, K.K. (1982). Effect of continuous application of fertilizer and manure on some physical properties of eroded alluvial soils. J Indian Soc Soil Sci., 30(1): 33-36.

Callan, N.W., Johanson, L.D., Westcott, P.M. and Welty, E.L. (2007). Herb and oil composition of dill (Anethum 
graveolens 304 Gurpreet Singh \& Sobaran Singh Impact Factor (JCC): 4.7987 NAAS Rating: 3.53 L.): Effect on crop maturity and plant density. Ind. Crop Prod., 282-287.

Darzi, M.T. and Haj Seyed Hadi, M. (2012). Effects of organic manure and nitrogen fixing bacteria on some essential oil components of coriander (Coriandrum sativum). International Journal of Agriculture and Crop Sciences. 4 (12): 787- 792.

Hellal, F.A., Mahfouz, S.A. and Hassan, F.A.S. (2011). Partial substitution of mineral nitrogen fertilizer by bio fertilizer on (Anethum graveolens L.) plant. Agriculture and Biology Journal of North America. 2 (4): 652- 660.

Kumar, A., Singh, R. and Chhillar, R.K. (2002). influence of irrigation and fertilizer levels on growth, seed yield and water use efficiency by Fennel (Foeniculum vulgare). Indian Journal of Agronomy, 47(2): 289-293.

Malhotra, S.K. and Vashishta, B.B. (2007). Response of Indian dill (Anethum sowa) and European dill (Anethum graveolens) varieties to different agro-techniques. Indian J. Agric. Sci., 77 (8): 43-46.

Meena, S. S., Mehta, R. S., Singh, R. K. and Vashishta, B. B. (2007). Effect of sheep manure, vermicompost and biofertilizers on productivity of dill (Anethum gravealens L.). Indian Journal of Arid Horticulture. 2 (2): 2930.

Selvrajan, M. and Chezhiyan, N. 2001. Effect of Azospirillum inoculation and nitrogen fertilization on growth and yield of coriander plant (Coriandrum sativum L.). South Indian horticulture, 49: 171-172.

Sharma, R.K., Dashora, S.L., Chaudhary, G.R., Agrawal, S., Jain, M.P. and D. Singh. 1996. In: P. Pundir and A.K. Dhama (Eds). Seed Spices research in rajasthan. Pub. Director Research. Raj. Agricultural university, Bikaner. P. 944.

Singh, A., Randhawa, G.S and Mahey, R.K. (1987). Oil content and yield of Dill (Anethium graveolens L) herb under some agronomic practices. Acta Horticulture, 208: 51-60. 12.

Singh, N. P., Sachan, R. S., Panday, P. C. and Bisht, P. S. (1999). Effect of a decade long fertilizer and manure application on soil fertility and productivity of rice wheat system on a Mollisol. J. Ind. Soc. Soil Sci., 47: 72-80.

Virmani, S. M. (1994).UNCED agenda 21: The new challenges for soils research. J. Indian Soc. Soil Sci. 42: 516-524. 14. Wani, S. P., and Lee, K. K. (1992) Role of bio-fertilizers in upland crop production. In: Fertilizers, organic manures, recyclable wastes and biofertilizers (H.L.S. Tandon, Ed.,) Pp. 91112. FDCO, New Delhi, India.

Wani, S.P., and Lee K.K. (1992). Role of biofertilizer in upland crop production In: Fertilizer, organic manures recyclable wastes and bio-fertilizers (H.L.S. Tandon, Ed.,) Pp. 91-112, FDCO, New Delhi, India.

\section{How to cite this article:}

Khushbu Kumari Gour, A.M. Patel, M.K. Sheshama, K.G. Vyas and Girraj Sharma. 2017. Effect of INM on Growth and Yield Attributes of Dill Seed (Anethum graveolens L.) Under North Gujarat Agro-Climatic Condition. Int.J.Curr.Microbiol.App.Sci. 6(12): 2587-2597. doi: https://doi.org/10.20546/ijcmas.2017.612.300 